\title{
Características clínicas e história familiar em pacientes ambulatoriais com transtorno obsessivo-compulsivo
}

\author{
Ygor Arzeno Ferrão* \\ Paulo Rogério Dalla Colletta de Aguiar** \\ Luciano Minuzzi*** \\ Rodrigo Grillo**** \\ Renan Lopes** \\ Rafael Rosa $a^{* * * *}$
}

\section{INTRODUÇÃO}

Após anos de estudos sobre a genética das principais síndromes psiquiátricas, sob diversos enfoques e objetivos, não restam dúvidas sobre a agregação familiar dos transtornos mentais, sobretudo do transtorno obsessivo-compulsivo (TOC). Os primeiros estudos que enfocaram a etiologia do TOC, e mesmo aqueles em que as primeiras descrições nosológicas foram feitas, já descreviam os aspectos de distribuição familiar da doença ${ }^{1}$. Estudos recentes mais

\footnotetext{
Trabalho realizado no Ambulatório do Serviço de Psiquiatria do Hospital Materno-Infantil Presidente Vargas. Este trabalho é resultado de dados parciais de projeto de pesquisa realizado pelo primeiro autor.

* Psiquiatra, Ambulatório de Transtornos do Espectro ObsessivoCompulsivo do Hospital Materno-Infantil Presidente Vargas, Porto Alegre RS. Doutor em Psiquiatria pela Universidade de São Paulo.

** Acadêmicos de Medicina, Fundação Faculdade Federal de Ciências Médicas de Porto Alegre, RS.

*** Psiquiatra, Universidade de Arhus, Dinamarca.

**** Psiquiatra, Ambulatório de Transtornos do Espectro ObsessivoCompulsivo do Hospital Materno-Infantil Presidente Vargas, Porto Alegre, RS.

***** Médico residente em Genética, Fundação Faculdade Federal de Ciências Médicas de Porto Alegre, RS.
}

criteriosos, em que os pesquisadores utilizaram entrevistas diagnósticas estruturadas de probandos e controles, confirmaram 0 componente familiar do $\mathrm{TOC}^{2}$.

Estudos de gêmeos indicam índices de concordância de TOC entre gêmeos monozigóticos que variam entre 53 e $87 \%$, enquanto que, entre dizigóticos, eles variam de 22 a $47 \%{ }^{3}$. Os dados obtidos em estudos com essa metodologia dão suporte à hipótese de que a hereditariedade está vinculada à etiologia do TOC. Em um estudo com 107 famílias de pacientes com TOC, foi sugerido que o modelo de transmissão genética compatível seria o autossômico dominante, com penetrância maior nas mulheres ${ }^{4}$, apesar de Nicolini et al., em estudo com 24 famílias de pacientes com TOC, terem concluído não ser possível a exclusão de um padrão autossômico recessivo ${ }^{5}$. Apesar da hipótese de maior penetrância genética em mulheres, o que estudos epidemiológicos evidenciam, até o momento, é uma prevalência igual entre os sexos.

Alguns estudos de segregação, como o de Alsobrook et al., concluem que, em famílias de pacientes com escores elevados de sintomas 
de simetria e ordenação, existe o provável envolvimento de um gene de efeito maior ${ }^{6}$. que se especula atualmente é que polimorfismos genéticos funcionais - como a disfunção de alelos das enzimas catecol-Ometiltransferase (COMT) e monoaminoxidaseA (MAO-A), além de alterações em genes dos sistemas serotonérgico e dopaminérgico estejam ligados à patogênese da doença ${ }^{7}$.

Estudos recentes avaliando a presença ou não do componente história familiar de TOC vêm sendo motivados por peculiaridades clínicas diversas, como a idade de início dos sintomas, o curso da doença, além de tipos, freqüência e gravidade de sintomas obsessivos e compulsivos. Os estudos tentam descrever subtipos de TOC com seus respectivos fatores de risco para uma resposta adequada ou não aos tratamentos convencionais ${ }^{8}$.

Apesar da complexidade da genética do TOC e das dúvidas que permanecem sobre seu padrão de transmissão, a constatação da ocorrência do transtorno em famílias nos permite especular sobre diferenças clínicas entre indivíduos com e sem história familiar de TOC e sobre a resposta às terapêuticas existentes. Com o objetivo de estudar a associação de características clínicas à presença ou não de história familiar de TOC, foi realizada uma revisão dos pacientes atendidos ambulatorialmente no Hospital Materno-Infantil Presidente Vargas de Porto Alegre (RS).

\section{MATERIAIS E MÉTODOS}

Dos 122 pacientes cadastrados, foram estudados retrospectivamente 111 pacientes do Ambulatório de Transtornos do Espectro Obsessivo-Compulsivo do Hospital MaternoInfantil Presidente Vargas, através de seus registros no protocolo de primeira consulta e seguimento ambulatorial, no período de julho de 1994 até julho de 2002. O critério de inclusão na pesquisa foi o diagnóstico de TOC de acordo com a Classificação Internacional de Doenças (CID-10). Desta forma, 11 pacientes foram excluídos do estudo por apresentar outros diagnósticos (três apresentavam jogo patológico e oito apresentavam tricotilomania e/ou auto-escoriação da pele sem comorbidade com TOC). Os cálculos estatísticos foram realizados, para um $\alpha=5 \%$, pelo teste de significância do qui-quadrado com fator de correção de Yates para o intervalo de confiança de $95 \%$ e análise de variância, através do programa SPSS 10.0.

$\mathrm{O}$ instrumento utilizado para a coleta de informações abordava questões como: data de nascimento, idade atual do paciente, gênero, etnia, idade de início dos sintomas, idade de início do tratamento para TOC, história familiar do paciente, lista de sintomas da Escala de Sintomas ObsessivoCompulsivos de Yale-Brown (YBOCS), escores da YBOCS, lista de psicofármacos e dosagens utilizadas, técnicas psicoterápicas realizadas, necessidade e número de internações hospitalares por causa do TOC e diagnósticos de eixo IV (fatores de risco sociais). A história familiar foi obtida através de entrevista semi-estruturada com o paciente e, quando possível, com um familiar.

\section{RESULTADOS}

Foram obtidas informações de 84 pacientes quanto à idade de início dos sintomas, sendo que $15(17,8 \%)$ apresentavam história familiar de TOC $(\mathrm{HF}+)$ e $69(82,2 \%)$ não apresentavam história familiar de TOC (HF-). A média de idade de início dos sintomas no grupo $\mathrm{HF}+$ foi menor, $17,8$ anos ( $D P=8,69)$, em comparação ao grupo HF-, cuja média foi de 20,8 anos (DP=9,65), diferença estatisticamente significativa $(p=<0,001)$. Oitenta e sete pacientes foram avaliados quanto à idade de início do tratamento do TOC, sendo 15 (17,2\%) com história familiar e $72(82,8 \%)$ sem história familiar de TOC. A média de idade de início do tratamento encontrada no grupo HF+ foi de 28,6 anos (DP= 12), média estatisticamente menor que a do grupo HF-, que foi de 30,6 anos ( $D P=11,6$ ) $(p=0,046)$. O grupo HF+ demorou, em média, 11,36 anos $(D P=11,5)$ para buscar auxílio profissional para a doença, ao passo que o grupo HF- levou, em média, 9,54 anos (DP=11), com um $p=0,067$.

Os pacientes em estudo foram avaliados quanto à freqüência dos diferentes tipos de obsessões do TOC. Os grupos não apresentaram diferenças significativas para nenhum tipo de obsessão nesta amostra.

Ambos os grupos foram também avaliados quanto à freqüência dos diferentes tipos de compulsões. Foi encontrada uma freqüência maior de colecionismo no grupo $\mathrm{HF}+: 4$ de 15 pacientes $(26,6 \%)$ no grupo $\mathrm{HF}+$ e 3 de 69 pacientes $(4,3 \%)$ no grupo HF-, diferença estatisticamente significativa $(p=0,029)$.

Não houve diferença relevante na variedade de fármacos utilizados no tratamento dos grupos estudados, nem na freqüência da aplicação da terapia cognitivo-comportamental.

A comparação da intensidade dos sintomas 
nos dois grupos, avaliada pela escala YBOCS, está descrita na Tabela 1.

Os grupos foram avaliados quanto à necessidade de outros tratamentos além de psicofarmacoterapia para o manejo e controle da sintomatologia do TOC. Houve diferença significativa entre os grupos, sendo que oito de 15 pacientes $(53,3 \%)$ com história familiar e 13 de 69 pacientes $(18,8 \%)$ sem história familiar necessitaram de terapia complementar à farmacológica (terapia cognitivo comportamental, grupoterapia, outras psicoterapias, técnicas de relaxamento, etc.) $(p=0,022)$.
Houve tendência de relevância estatística quanto ao número de pacientes que necessitaram de internação hospitalar no curso do tratamento. Dez de 17 pacientes $(58,8 \%)$ foram internados em algum momento de seu tratamento no grupo $\mathrm{HF}_{+}$, ao passo que 26 de 75 (34,6\%) necessitaram de internação no grupo HF- $(p=0,065)$. Não houve diferença estatística quanto ao número médio de internações entre os grupos.

Não foram encontradas diferenças relevantes quanto aos diagnósticos de eixo IV (problemas sociais e fatores de estresse desencadeantes) entre os grupos em estudo.

Tabela 1 - Comparação da intensidade dos sintomas obsessivo-compulsivos entre os grupos com e sem história familiar de TOC

\begin{tabular}{lcccc}
\hline \multicolumn{1}{c}{ YBOCS } & $\begin{array}{c}\text { História familiar positiva } \\
(\mathbf{n = 1 3 )} \\
\text { média (DP) }\end{array}$ & $\begin{array}{c}\text { História familiar negativa } \\
(\mathbf{n = 3 8 )} \\
\text { média (DP) }\end{array}$ & $\mathbf{F}$ & $\mathbf{p}$ \\
\hline Subescala de obsessões & $10,46(3,73)$ & $8,61(4,89)$ & 5,73 & 0,023 \\
Subescala de compulsões $^{\text {Escore total }}{ }^{*}$ & $11,77(2,86)$ & $9,24(5,04)$ & 13,76 & $<0,001$ \\
\hline
\end{tabular}

*O escore total da YBOCS foi encontrado em 14 prontuários de pacientes com história familiar positiva de TOC e em 43 prontuários de pacientes com história familiar negativa de TOC.

YBOCS = Escala de Sintomas Obsessivo-Compulsivos de Yale-Brown; DP = desvio padrão; $n=$ tamanho amostral.

\section{DISCUSSÃO}

Sendo um estudo observacional, as limitações da metodologia retrospectiva por análise de prontuários, como o número de perdas para cada variável e a confiabilidade das informações, por exemplo, além da amostra ser de pacientes referendados a um serviço de referência para TOC, podem ter influenciado alguns resultados, devendo-se, pois, atentar para possíveis erros na generalização dos achados. Contudo, as restrições metodológicas não nos impedem de observar interessantes aspectos concernentes à ocorrência de história familiar em pacientes com TOC.

Nossa amostra evidenciou $17,8 \%$ dos pacientes com TOC como tendo história familiar positiva para o transtorno, o que está relativamente de acordo com as estimativas da literatura, como evidenciam os estudos mencionados a seguir.

No primeiro estudo realizado com famílias de pacientes com TOC na América Latina, Nicolini et al. investigaram 268 parentes em $1^{\circ}$ probandos com TOC. Trinta por cento daqueles pacientes apresentavam história familiar positiva para TOC. Entre os parentes em 1 응 grau, a freqüência de TOC foi de $4,85 \%$, e entre os parentes de $2^{\circ}$ grau, de $2,13 \%$. Assim como em nossa amostra, os probandos com história familiar positiva do estudo de Nicolini et al. também apresentavam idade de início dos sintomas menor do que os probandos sem história familiar de TOC $^{9}$.

Pauls et al. estudaram, com entrevistas psiquiátricas estruturadas, parentes em $1 \stackrel{0}{ }$ grau de 100 probandos com TOC $(n=466)$ e 133 parentes (controles) em $1^{\circ}$ grau de 33 indivíduos sadios. Observaram $10,9 \%$ de TOC e $7,9 \%$ de TOC subclínico nos parentes em $1^{\circ}$ grau dos probandos. Entre os controles, as taxas foram de $1,9 \%$ de TOC e $2,0 \%$ de TOC subclínico ${ }^{10}$.

Com efeito, pacientes com história familiar de TOC apresentaram, em nosso estudo, o início dos seus sintomas em idade mais remota, mas levaram em média 2 anos a mais do que os pacientes sem história familiar para procurar ajuda médica. Disso pode-se inferir que o grupo com carga genética positiva apresentaria uma 
predisposição maior para desenvolver TOC, desde que os fatores ambientais não consigam inibir essas manifestações.

Além de se manifestarem mais precocemente nos pacientes com história familiar positiva, os sintomas tendem a ser mais intensos, como fica evidente em nossa amostra pelos escores mais altos na escala YBOCS, pela necessidade de maior variedade de métodos terapêuticos e pela maior necessidade de internações hospitalares. Essa diferença clara entre os grupos estudados pode indicar que a história familiar de TOC é um fator de risco para maior gravidade ou complexidade dos casos.

Corroborando esses achados, mais recentemente, Gonzáles, ao comparar uma amostra constituída por 173 parentes em $1^{\circ}$ grau de pacientes com diagnóstico de TOC, inclusive de crianças com idade igual ou superior a 6 anos, com 348 parentes em $1^{\circ}$ grau de 42 pacientes de um serviço ambulatorial de otorrinolaringologia, concluiu, entre outras coisas, que: 1) TOC e sintomas obsessivocompulsivos são condições que se agregam em famílias; 2) os transtornos de tique, transtorno dismórfico corporal e transtorno de compulsão alimentar periódico são mais prevalentes entre familiares de pacientes com TOC do que entre controles e parecem fazer parte de um espectro familial do TOC; 3) parentes de pacientes com TOC de início precoce apresentam maior risco de desenvolver TOC, sintomas obsessivocompulsivos, transtornos de tique e a somatória dos transtornos estudados do que os parentes de pacientes com TOC de início tardio ${ }^{11}$.

Os autores sugerem que o maior tempo para buscar tratamento nas famílias com história familiar positiva pode dever-se ao fenômeno de acomodação familiar, definido como a participação da família nos comportamentos associados aos rituais dos pacientes e a modificação nas rotinas diárias da família. A acomodação familiar constitui-se de aspectos como: participação dos familiares nos sintomas obsessivo-compulsivos; modificações do funcionamento familiar (hábitos, lazer, trabalho, etc.) como conseqüência direta dos sintomas; desgaste emocional e psíquico que os sintomas do paciente ocasionam; e conseqüências da nãoparticipação dos familiares nos sintomas ${ }^{12}$. Nas famílias com história positiva, o aprendizado, por experiência prévia, da acomodação familiar pode fazer com que os familiares tolerem os sintomas obsessivo-compulsivos. É interessante ressaltar que, em condições clínicas (hipertensão arterial sistêmica, diabete melito), a acomodação familiar mais freqüentemente não ocorre, fazendo-nos crer que os transtornos psiquiátricos (dentre eles, o TOC) estejam mais propensos a este fenômeno.

A literatura sugere que o colecionismo pode indicar um subtipo específico de TOC, o que está em concordância com os resultados de nossa amostra ${ }^{13,14}$. Moreno et al. relatam, por exemplo, que o colecionismo é um fator de risco para mau prognóstico ${ }^{15}$. Na realidade, há escassez, na literatura, de estudos com metodologia e tamanhos amostrais adequados que evidenciem a associação de colecionismo e herança genética do TOC.

\section{CONSIDERAÇÕES FINAIS}

Em resumo, os pacientes portadores de TOC com história familiar positiva, em nosso estudo, mostraram idade de início dos sintomas mais precoce, levando mais tempo para buscar ajuda adequada. Além disso, evidenciaram maior gravidade dos sintomas medidos pela YBOCS e maior complexidade terapêutica. Isso, associado ao fato de não ter havido significância estatística na comparação quanto aos fatores sociais de estresse (eixo IV), reforça os achados da literatura de que o TOC possui constituintes biológicos em sua patogênese, ou que pelo menos um subtipo de TOC parece possuir uma influência biológica mais evidente. Ainda que isso ocorra, a influência ambiental ganha força na manutenção dos sintomas, quando o grupo com história familiar positiva leva mais tempo para procurar tratamento adequado, o que hipotetiza o papel da acomodação familiar como agravante. Iniciativas como as de associações de familiares e pacientes na instrução e educação sobre o TOC são imprescindíveis na ajuda ao tratamento dos pacientes.

A subtipagem do TOC usando-se vários conteúdos das obsessões e compulsões ainda é um desafio que promete revelar aspectos importantes no entendimento da doença e no planejamento terapêutico. Uma tentativa nesse sentido vem sendo realizada pelo grupo da Universidade de Yale, com colaboração da Universidade de São Paulo, que está elaborando uma nova escala YBOCS baseada nos subgrupos de sintomas encontrados no trabalho de Leckman et al. ${ }^{16}$.

Assim é que, ainda que com as limitações do estudo (pequeno número de pacientes, método retrospectivo com dados de prontuário, com perdas importantes de informações), 
podemos concluir que a história familiar de TOC em pacientes portadores de TOC pode ser um fator de risco para maior gravidade e complexidade dos sintomas. Estudos prospectivos, com informações sobre história familiar de TOC e transtornos psiquiátricos colhidas diretamente com pacientes e familiares, além da inclusão de entrevistadores cegos para a condição, poderão evidenciar mais adequadamente esses resultados.

\section{REFERÊNCIAS BIBLIOGRÁFICAS}

1. Pitman RK. Pierre Janet on obsessive-compulsive disorder (1903). Review and commentary. Arch Gen Psychiatry 1987;44:226-32.

2. Hettema JM, Neale MC, Kendler KS. A review and metaanalysis of the genetic epidemiology of anxiety disorders. Am J Psychiatry 2001;158:1568-78.

3. Rasmussen AS, Tsuang MT. Clinical characteristics and family history in DSM-III obsessive-compulsive disorder. Am J Psychiatry 1986;143:317-22.

4. Cavalini MC, Pasquale L, Bellodi L, Smeraldi E. Complex segregation analysis for obsessive-compulsive disorder and related disorders. Am J Med Genet 1999;88:38-43.

5. Nicolini H, Hanna G, Baxter L, Schwartz J, Weissbacker K, Spence MA. Segregation analysis of obsessivecompulsive disorder and related disorders. Preliminary results. Ursus Med 1991;1:25-8.

6. Alsobrook JP, Leckman JF, Goodman WK, Rasmussen AS, Pauls DL. Segregation analysis of obsessivecompulsive disorder using symptom-based factor scores. Am J Med Genet 1999;88:669-75.

7. Pato MT, Schindler KM, Pato CN. The genetics of obsessive-compulsive disorder. Curr Psychiatry Rep 2001;3:163-8.

8. Bebbington PE. Epidemiology of obsessive-compulsive disorder. Br J Psychiatry 1998;35(suppl):2-6.

9. Nicolini H, Weissbecker K, Mejia JM, Sanchez de Carmona M. Family study of obsessive-compulsive disorder in a Mexican population. Arch Med Res 1993;24(2):193-8.

10. Pauls DL, Alsobrook JP 2nd, Goodman W, Rasmussen $\mathrm{S}$, Leckman JF. A family study of obsessive-compulsive disorder. Am J Psychiatry 1995;152:76-84.

11. Gonzales C. Estudo de famílias no transtorno obsessivocompulsivo [tese]. São Paulo: Universidade Federal de São Paulo; 2003.

12. Guedes ML. Transtorno obsessivo-compulsivo: um estudo do processo de acomodação familiar [dissertação]. São Paulo: Universidade Federal de São Paulo; 1997. 80pp.

13. Baer L. Factor analysis of symptom subtypes of obsessive compulsive disorder and their relation to personality and tic disorders. J Clin Psychiatry 1994;55(Suppl):18-23

14. Zhang $H$, Leckman JF, Pauls DL, Tsai CP, Kidd KK, Campos MR. Genome wide scan of hoarding in sib pairs in which both sibs have Gilles de la Tourette syndrome. Am J Hum Genet 2002;70(4):896-904.

15. Moreno AC, Calo JJP, Pinero MV, Zaragoza CL, Navarro VF, Marsa MD. Evolución y respuesta terapéutica en el trastorno obsesivo-compulsivo. Actas Luso Esp Neurol Psiquiatr Cienc Afines 1995;23(1):9-19.

16. Leckman JF, Grice DE, Boardman J, Zhang H, Vitale A, Bondi C, et al. Symptoms of obsessive-compulsive disorder. Am J Psychiatry 1997;154(7):911-7.

\section{RESUMO}

Introdução: A ocorrência familiar do transtorno obsessivo-compulsivo permite-nos especular sobre diferenças clínicas entre indivíduos com história familiar de TOC $(\mathrm{HF}+)$ e sem história familiar de TOC (HF-). Este estudo investigou a associação entre história familiar de TOC e algumas características clínicas. Material e métodos: Foram estudados retrospectivamente 111 pacientes através de revisão de prontuários de atendimento no Hospital MaternoInfantil Presidente Vargas de Porto Alegre, no período de julho de 1994 até julho de 2002. Resultados: $A$ idade média de início dos sintomas foi menor no grupo $H F+(17,8$ anos $\pm 8,69$ versus 20,8 anos $\pm 9,65$; $p=0,000963)$. A intensidade dos sintomas obsessivocompulsivos medidos pela YBOCS foi mais grave no grupo $H F+(22,5 \pm 4,16$ versus $17,93 \pm 9,95 ; p<0,001)$. Encontrou-se uma incidência maior de colecionismo no grupo $H F+: 4$ de 15 pacientes $(26,6 \%)$ versus 3 de 69 pacientes (4,3\%); $p=0,029$. Não houve diferença relevante na variedade de fármacos utilizados no tratamento dos grupos estudados, bem como na freqüência da aplicação da terapia cognitivocomportamental. Oito de 15 pacientes $(53,3 \%) \mathrm{com}$ história familiar e 13 de 69 pacientes (18,8\%) sem história familiar necessitaram de terapia complementar à farmacológica $(p=0,022)$. Conclusão: Em resumo, os pacientes portadores de TOC com história familiar positiva para o mesmo, em nosso estudo, mostraram início dos sintomas mais precoce, maior gravidade dos sintomas medidos pela YBOCS e maior complexidade terapêutica, além de presença mais freqüente de colecionismo. Estudos prospectivos, com informações colhidas diretamente dos pacientes e dos familiares, além da inclusão de grupos-controle randomizados, poderão evidenciar adequadamente esses resultados.

Descritores: Transtorno obsessivo-compulsivo, história familiar, colecionismo.

\section{ABSTRACT}

Introduction: The occurrence of obsessivecompulsive disorder (OCD) in families suggests the existence of clinical differences between patients with $(\mathrm{FH}+)$ and without $(\mathrm{FH}-)$ a family history of OCD. This study aims at assessing the association between family history of OCD and some clinical features. Material and methods: 111 patients were retrospectively studied (review of medical records) at the outpatient clinic at Hospital Materno-Infantil Presidente Vargas, in Porto Alegre, Brazil, between July 1994 and July 2002. Results: Mean age at onset of symptoms was lower in the $\mathrm{FH}+$ group (17.8 years \pm 8.69 vs. 20.8 years \pm 9.65; $p=0.000963)$. The severity of obsessive-compulsive symptoms (YBOCS) was higher in the $\mathrm{FH}+$ group $(22.5 \pm 4.16$ vs. $17.93 \pm$ 9.95; $p<0.001)$. Hoarding was also more frequently found in the FH+ group: 4 of 15 patients (26.6\%) vs. 3 
of 69 patients (4.3\%); $p=0.029$. We found no significant difference in the variety of drugs used in the treatment of the two groups, as well as in the frequency of application of the cognitive-behavioral therapy. Eight of 15 patients (53.3\%) with a family history and 13 of 69 patients (18.8\%) without a family history of OCD had to complement the psychopharmacological therapy $(p=0.022)$. Conclusion: In our study, OCD patients with a positive family history of the disease showed earlier onset of symptoms, more severe symptoms according to YBOCS and the need for a more complex treatment scheme. Hoarding was also more frequent in this group. Prospective studies, with information collected directly from patients and their families, as well as the inclusion of randomized control groups, are necessary to further confirm these results.

Keywords: Obsessive-compulsive disorder, family history, hoarding.

Title: Clinical features and family history in obsessivecompulsive outpatients

\section{RESUMEN}

Introducción: La ocurrencia familiar del trastorno obsesivo-compulsivo nos permite conjeturar sobre diferencias clínicas entre individuos con historia familiar de TOC $(\mathrm{HF+}$ ) y sin historia familiar de TOC (HF-). Este estudio investigó la asociación entre historia familiar de TOC y algunas características clínicas. Material y métodos: Se estudió retrospectivamente a 111 pacientes a través de revisión de prontuarios de atención en el Hospital Materno-Infantil Presidente Vargas, de Porto Alegre, en el periodo de julio de 1994 hasta julio de 2002. Resultados: La edad media de inicio de los síntomas fue menor en el grupo $\mathrm{HF}+(17,8$ años $\pm 8,69$ versus
20,8 años $\pm 9,65 ; p=0,000963)$. La intensidad de los síntomas obsesivo-compulsivos medidos por $Y$-BOCS fue más grave en el grupo $H F+(22,5 \pm 4,16$ versus $17,93 \pm 9,95 ; p<0,001)$. Hubo una incidencia mayor de coleccionismo en el grupo HF+: 4 de 15 pacientes (26.6\%) versus 3 de 69 pacientes (4,3\%); $p=0,029$. No hubo diferencia relevante en la variedad de fármacos utilizados en el tratamiento de los grupos estudiados, así como en la frecuencia de la aplicación de la terapia cognitivo-comportamental. Ocho de 15 pacientes $(53,3 \%)$ con historia familiar y 13 de 69 pacientes $(18,8 \%)$ sin historia familiar necesitaron de terapia complementar a la farmacológica $(p=0,022)$. Conclusión: En resumen, los pacientes portadores de TOC con historia familiar positiva para el mismo, en nuestro estudio, mostraron inicio más precoz de los síntomas, mayor gravedad de los síntomas medidos por Y-BOCS y mayor complejidad terapéutica, además de la presencia más frecuente de coleccionismo. Estudios prospectivos, con informaciones recogidas directamente de los pacientes y de los familiares, además de la inclusión de grupos de control randomizados, podrán evidenciar adecuadamente esos resultados.

Palabras clave: Trastorno obsesivo compulsivo, historia familiar, coleccionismo.

Título: Características clínicas e historia familiar en pacientes ambulatoriales con trastorno obsesivocompulsivo

Correspondência:

Ygor Arzeno Ferrão

Rua Padre Chagas, 185/403

CEP 90570-080 - Porto Alegre - RS

E-mail:ygoraf@terra.com.br

Copyright (C) Revista de Psiquiatria

do Rio Grande do Sul - SPRS 